\title{
Les « Comices viticoles » de Passage du poète: Ramuz, Flaubert et le discours médiocre
}

\author{
Valérie BucheLI \\ Université de Genève
}

\begin{abstract}
Une comparaison entre le fameux discours de Lieuvain, à l'occasion des Comices agricoles de Madame Bovary, et deux allocutions prononcées dans Passage du poète de Charles Ferdinand Ramuz, lors d'une scène que l'on pourrait voir comme des "Comices viticoles ", donne l'occasion de montrer, au-delà des ressemblances de surface entre les deux romans, l'attitude très distincte de chacun des auteurs devant de telles manifestations du langage ordinaire. Alors que Flaubert, comme on le sait, dévalue les «discours médiocres ", Ramuz procède de son côté à une véritable valorisation du lieu commun, auquel il attribue le pouvoir de réunir la collectivité villageoise autour d'une morale partagée.
\end{abstract}

Keywords : Ramuz, Flaubert, intertextualité, médiocrité, communauté

De la fameuse scène des Comices agricoles, dans Madame Bovary ${ }^{1}$, on pourrait dire qu'elle constitue le point ultime d'un travail de sape du langage, dont la critique a pu montrer qu'il était opéré constamment par Flaubert. Dominique Rabaté affirme par exemple que, au sein de ce roman, «l'effacement apparent de la parole de l'auteur au profit de celle de ses personnages ménage $[. .$.$] un arrière-plan ironique qui donne à entendre le ridicule$ et la pauvreté de nos discours $»^{2}$. Le commentateur appelle ainsi « discours médiocres » les propos tenus par les personnages, en une formule intéressante parce que relativement ambiguë, et il relève que le procédé passe par ce qu'il appelle «la dévaluation de l'autorité de la parole "*. Dans la longue harangue du représentant des notables, comme dans les platitudes philosophiques que Rodolphe sert à Emma, s'entremêlent en effet des formules attendues dont on aurait envie de vérifier la présence dans le Dictionnaire des idées reçues. Chez Flaubert, le lieu commun constitue bien un «lieu vain » - car ce n'est évidemment pas sans raison que son exubérant orateur porte ce nom, Lieuvain : le personnage devient ainsi l'incarnation même de ce discours inassignable et banal, marqué à proprement parler de vanité aux

I Il s'agit du chapitre VIII de la deuxième partie du roman. Voir Gustave Flaubert, Madame Bovary, in Euvres complètes, 3 vol., éd. Claudine Gothot-Mersch, Paris, Gallimard, « Bibliothèque de la Pléiade ", 200I-2013, t. III, pp. 266-286. Dorénavant, les références à ce roman seront indiquées par le sigle MB et placées entre parenthèses dans le texte.

2 Dominique Rabaté, « Réalisme et ironie. Les voies de la parole dans Dubliners », Littérature, 94, 1994, p. 38.

3 Ibid. 
deux sens du terme, de prétention comme de frivolité. Ainsi, dans l'œuvre flaubertienne, le "médiocre » au sens sociologique (" qui est de condition sociale moyenne ", dit le Trésor de la langue française) devient-il porteur de toute une axiologie négative.

Dans la perspective de redonner dans cette étude une valeur au lieu commun, j'aimerais examiner une scène toute parallèle à celle des Comices, dans un roman très différent - dans Passage du poète de Charles Ferdinand Ramuz, qui date de I923 - pour montrer d'un côté que l'auteur, à l'évidence, fait très consciemment référence à son prédécesseur, qu'il procède à un véritable jeu intertextuel avec le chapitre de Madame Bovary ; mais que, d'un autre côté, le contraste créé par le rapprochement des deux textes montre un traitement tout différent du « discours médiocre » et du « lieu commun » chez l'écrivain romand. Si Flaubert s'en sert sans doute pour miner tout le prestige du langage, comme le montre Rabaté, il me semble que l'intention est, pour ainsi dire, inverse de la part de Ramuz. Quant à lui, il élaborerait au contraire un système discursif au sein duquel lieu commun et discours médiocre sont valorisés, et même dotés de la valeur la plus haute possible, afin de leur faire supporter tout le poids de son éthique humaniste et terrienne.

\section{Flaubert : la haine du commun, du trivial, du médiocre}

Le rappel de quelques considérations sur les Comices agricoles de $\mathrm{Ma}$ dame Bovary, déjà mises en évidence par la critique, permettra d'observer ensuite dans Passage du poète les caractéristiques les plus dignes d'intérêt. On sait tout d'abord - l'expression est fameuse - que Flaubert a qualifié plusieurs fois la scène des Comices de «symphonie »", parce qu'elle présente une " alternance savante des plans, des dialogues et des discours, [...] des lieux communs politiques et sentimentaux $"$. Mais - il faut le préciser aussitôt - c'est une symphonie qui se joue sur fond de rivalité entre les deux discours, en une musicalité condescendante, si l'on peut dire. La proclamation officielle et le monologue galant sont en concurrence : lorsque tout à coup «la voix du Conseiller s'él[ève] d'un ton extraordinaire» (MB, 275), Rodolphe

4 On trouve au moins deux occurrences du terme ou de ses composés dans la correspondance : «J'ai bien peur que mes comices ne soient trop longs. C'est un dur endroit. J'y ai tous mes personnages de mon livre en action et en dialogue, les uns mêlés aux autres, et par là-dessus un grand paysage qui les enveloppe. Mais, si je réussis, ce sera bien symphonique " (Gustave Flaubert, lettre à Louise Colet, 7 septembre I853, in Correspondance, 5 vol., éd. Jean Bruneau, Paris, Gallimard, «Bibliothèque de la Pléiade», 1973-2007, t. II, p. 426); et : « Si jamais les effets d'une symphonie ont été reportés dans un livre, ce sera là. Il faut que ça hurle par l'ensemble, qu'on entende à la fois des beuglements de taureaux, des soupirs d'amour et des phrases d'administrateurs » (Flaubert, lettre à Louise Colet, I2 octobre I853, ibid., p. 449).

5 Jeanne Goldin, Les Comices agricoles de Gustave Flaubert. Transcription intégrale et genèse dans le manuscrit g 223, 2 vol., Genève, Droz, 1984, t. I, «Étude génétique », p. I6. 
doit s'interrompre ; au contraire, quand Lieuvain évoque « la pratique des devoirs ", le courtisan lui répond indirectement pour le contester avec hauteur, disant qu'il est « assommé de ces mots-là » et enchaînant à son tour sur " le devoir ", au singulier, qui serait « de sentir ce qui est grand, de chérir ce qui est beau, et non pas d'accepter toutes les conventions de la société, avec les ignominies qu'elle nous impose » $(\mathrm{MB}, 277)$ - en un refrain tout aussi cliché que le propos duquel il prétend se distinguer. Les deux hommes rivalisent effectivement de « discours médiocres » pour prendre l'ascendant sur leur public, en deux paroles dont le « ridicule » et la « pauvreté » se reflètent l'une dans l'autre. Quelques mots de Nathalie Sarraute condensent admirablement toutes ces remarques :

Les lieux communs débités par Rodolphe, son raffinement de mauvais goût, sa fausse sincérité, son mépris satisfait et dédaigneux des convenances, ses sentiments de pacotille se détachent, rehaussés, sur la platitude plus grossière des discours officiels, dont les clichés à leur tour sont admirablement mis en valeur par les déclarations amoureuses et les considérations philosophiques de Rodolphe ${ }^{6}$.

Cette division des deux intervenants est redoublée, à un second niveau, par la position très surplombante du narrateur flaubertien par rapport à ses personnages. On peut s'amuser à relever, dans la déclamation du Conseiller, toute une série de stupidités plaisantes qui témoignent de la maîtrise, par Flaubert, des dérapages rhétoriques de son orateur. À force de multiplier les images conventionnelles, Lieuvain en vient par exemple à présenter le monarque comme dirigeant « le char de l'État parmi les périls incessants d'une mer orageuse » $(\mathrm{MB}, 275)$, en un pataquès métaphorique particulièrement grotesque. Rodolphe pour sa part est sans doute moins bête, mais l'auteur s'amuse néanmoins à placer dans sa bouche les poncifs les plus ressassés sur les « âmes sans cesse tourmentées ", à qui il faut « tour à tour le rêve et l'action, les passions les plus pures, les jouissances les plus furieuses » $(\mathrm{MB}, 276)$.

Un étagement caractéristique se révèle ainsi, avec les bourgeois du village tout en bas, puis, au-dessus, Rodolphe méprisant les bourgeois depuis sa fenêtre, et enfin Flaubert lui-même qui juge tacitement Rodolphe à son tour - dénonçant par là la vanité de ses prises de parole ${ }^{7}$. Parlant de la rédaction des Comices à Louise Colet, l'écrivain use d'ailleurs des termes mêmes

6 Nathalie Sarraute, Paul Valéry et l'Enfant d'Éléphant suivi de Flaubert le précurseur, Paris, Gallimard, 1986, p. 82.

7 Avec le mépris affiché par Mme Lefrançois, l'aubergiste de Yonville, pour les "vachers » et les «va-nu-pieds » que deviennent dans sa bouche les paysans fêtés au cours des comices $(\mathrm{MB}, 267)$, la stratification sociale acquiert même un niveau supplémentaire - mais ceux-ci, à l'image de l'emblématique Catherine Leroux, «ce demi-siècle de servitude » (MB, 282-283), ne parlent pas dans les romans de Flaubert. 
dans lesquels la critique décrit aujourd'hui le langage qu'il y met en scène :

Ce à quoi je me heurte, c'est à des situations communes et un dialogue trivial. Bien écrire le médiocre et faire qu'il garde en même temps son aspect, sa coupe, ses mots même, cela est vraiment diabolique, et je vois se défiler maintenant devant moi de ces gentillesses en perspective pendant trente pages au moins ${ }^{8}$.

Le regard sans concession de Flaubert est donc mis au service d'un dégoût général de la petite bourgeoisie, qu'il exprime partout dans sa correspondance pendant cette période.

\section{Ramuz : la « valeur du lieu commun »}

Ramuz, quant à lui, ne s'est pas montré très prolixe au sujet de l'œuvre flaubertienne. Lorsqu'il parle de son prédécesseur, il fait cependant référence, en quelques occasions, à une proximité qu'il perçoit entre leurs deux styles. Le 20 décembre I9OI, il évoque par exemple dans son Journal, très rapidement, un " parallèle entre [s] on besoin de rythme léger, libre et tout d'oreille et le merveilleux rythme des phrases de Flaubert ", notant alors sa "grande envie de Madame Bovary "'. Je ne sais pas si, à l'inverse, il a jamais évoqué une distance idéologique qu'il pourrait y avoir entre l'ermite de Croisset et lui, mais cette distance me paraît d'autant plus sensible quand on l'observe, justement, sur un fond de ressemblance.

Le chapitre I4 de Passage du poète, l'avant-dernier du bref roman, constitue un exemple important de ce point de vue, parce qu'il présente une situation narrative tout à fait semblable à celle des Comices agricoles ${ }^{10}:$ c'est là encore l'évocation d'une "fête rustico-municipale ", comme dit Flaubert dans sa correspondance ${ }^{\mathrm{II}}$, pendant laquelle a lieu un rassemblement de la petite communauté fictive. Quelques notables montent alors sur une estrade tout identique à celle de Yonville pour louer, comme Lieuvain, les valeurs de la terre et les qualités de force et de patience des agriculteurs, qui sont dans ce cas des vignerons. Le substantif qui désigne l'assemblée chez Flaubert n'est jamais employé par Ramuz - il ne va pas jusque-là ; mais je suggère de nommer cette scène les «Comices viticoles » de Passage du poète. Lors de la description de la fête sont rapportées deux allocutions successives, dans

8 Flaubert, lettre à Louise Colet, I2 septembre I853, in Correspondance, op. cit., t. II, p. 429.

9 Charles Ferdinand Ramuz, Journal, 3 vol., Genève, Slatkine, 2005, t. I, p. 244.

Io Voir id., Passage du poète, in Romans, 2 vol., éd. Doris Jakubec, Paris, Gallimard, « Bibliothèque de la Pléiade ", 2005, t. II, pp. 303-306. Dorénavant, les références à ce roman seront indiquées par le sigle PP et placées entre parenthèses dans le texte.

II Flaubert, lettre à Louise Colet, 15 juillet I853, in Correspondance, op. cit., t. II, p. 386. 
chacune desquelles deux niveaux de voix se font entendre, puisque deux individus emblématiques du village se manifestent pour les entrecouper, sur le modèle des interventions de Rodolphe. Ce n'est donc pas sans raison que Ramuz évoque une véritable " cérémonie ", dans le paragraphe introducteur de la séquence narrative (PP, 303), usant ainsi d'un terme qui dit la tournure solennelle ${ }^{\mathrm{I}}$, plus ou moins sacrée, de la fête - de même que son caractère conventionnel.

Mais la comparaison entre les deux scènes romanesques conduit également, bien sûr, à souligner leurs différences. La fonction des deux niveaux de voix distincts, qui tend à un échange authentique entre l'estrade et le public populaire, comme le statut du narrateur, équivalent à celui des personnages, éloignent en effet fondamentalement la conception que Ramuz se fait de l'homme de celle qui est portée par Madame Bovary, permettant ainsi d'illustrer une valorisation du lieu commun à l'œuvre dans Passage du poète. Si la forme conférée aux discours, d'un roman à l'autre, est semblable, leur fonction n'en est pas moins très éloignée.

Un simple coup d'œil au chapitre permet de voir que, pour rédiger sa propre scène de comices, Ramuz utilise le même procédé que Flaubert : dès le premier discours, il orchestre bien deux paroles indépendantes, celle d'un orateur anonyme, dont le statut exact demeure assez indéfini, en italiques, et celle d'un villageois, le dénommé Bovard. Bovard, dans le roman, n'est pas n'importe quel personnage : il représente celui des vignerons non seulement qui est personnifié, qui se détache de la masse anonyme, mais encore qui, sous l'influence du poète du titre, va peu à peu trouver les mots pour transmettre son ressenti et son expérience, celui qui finit par trouver à s'exprimer alors même que les mots, au départ, lui manquaient. À partir du moment où " le poète est venu », comme le dit le texte, « les mots sortent de lui tout le temps, comme quand les ruches se réveillent » (PP, 257) ; irrépressiblement, il se met alors à communiquer. Dans la scène, l'allocution officielle est donc interrompue par les interjections de ce personnage, qui tronçonnent le discours et le recouvrent par moments brièvement.

Bovard - on le voit sitôt qu'on pénètre plus avant dans le texte - ne se comporte pourtant pas du tout comme Rodolphe : au lieu de se montrer supérieur à l'orateur, il lui témoigne son accord complet, si bien que ses interventions marquent sa participation et son enthousiasme. Quand il s'immisce pour la première fois dans le discours, il s'exclame : "C'est vrai " (PP, 304) ; et, lors de sa deuxième acclamation, il est encore plus emporté, puisqu'il « crie » longuement : «Ah ! c'est vrai, c'est vrai, c'est très vrai ! [...] Et c'est comme j'ai toujours dit » (PP, 305). Cette correspondance relevée par le personnage n'est pas qu'une formule : effectivement, les mots de l'orateur

I2 Flaubert, quant à lui, nomme ironiquement les Comices « la solennité » (MB, 266). 
peuvent être mis en relation avec certaines phrases énoncées par Bovard, plus tôt dans le roman. Dans une grande scène du chapitre 7 , il tient en effet un long discours, pour lui-même, quant à sa déontologie de paysan, à son inscription dans une tradition millénaire et au sens des tâches exigeantes qu'il accomplit ; et, alors, ses déclarations particulières sont de fait très similaires aux propos tenus lors de la cérémonie des comices. Tandis que l'orateur affirme de manière générale, à propos de la terre et des vignes : "ça se met à vous obéir, ça se plie à vous, ça devient docile », Bovard prétendait déjà, en contemplant ses ceps : "C'est que c'est tout plié à nous, par ici » (PP, 256). Un tel rapprochement entre les deux paroles nous en apprend beaucoup, parce que la même idée est énoncée par les deux hommes, celle d'une docilité de la terre qui se met à « obéir » aux vœux des hommes, au point qu'une tournure identique est employée dans les deux tirades. L'un et l'autre intervenants usent en effet de la construction plier (quelque chose à quelqu'un), qui est certes attestée dans la langue littéraire et chez des écrivains français, mais qui prend, chez Ramuz, une allure très matérielle, presque charnelle, qui lui est propre et qui rejoint un parler régional et familier.

Si Bovard tombe aussi complètement d'accord avec le représentant des autorités, ce n'est pas en vertu d'une entente particulière qu'il y aurait entre eux deux ; c'est bien plutôt parce que leur discours est composé essentiellement de lieux communs. Leur complicité est possible du fait même que leurs deux manières de s'exprimer sont basées sur des tournures et des représentations partagées, qui sont usées par l'habitude - qui sont, elles aussi, pliées à eux. Il suffira de citer comme exemple l'aspect assez fruste des considérations psychologiques quant à ce qui fait la satisfaction des vignerons, dans la tirade qui suit la seconde interruption de Bovard : "... la femme va bien, les enfants vont bien, la cuisine est propre; alors les hommes se rapprochent, parce que quand l'homme est content il va à l'homme, et l'homme qui n'est pas content fuit l'homme » (PP, 305). Le style est ici d'une simplicité enfantine, depuis la parataxe des trois premières séquences, avec le lexique qu'elles emploient (la formule scandée va bien / vont bien, la mention de « la cuisine », les articles définis à fonction généralisante), jusqu'à l'expression de la consécution avec " alors », à l'égalisation de l'homme individuel et des hommes avec le même terme repris cinq fois et au balancement content / pas content qui réduit à une alternative naïve la gamme des sentiments humains. Mais il n'empêche qu'un tel dépouillement prosaïque satisfait tout le monde, puisque se font entendre à ce moment les « applaudissements » du public ( $\mathrm{PP}, 305)$. La valeur de ces phrases élémentaires est donc reconnue par ceux mêmes à qui elles sont adressées, et l'acquiescement de Bovard s'élargit à un cercle beaucoup plus large.

Personne ne songe en effet à retenir contre l'orateur les maladresses rhétoriques de son discours, puisque tous les partagent. D'un point de vue sty- 
listique (avec des insistances à la fois très efficaces et très familières, comme " cette terre, il faut d'abord avoir sué contre, avoir fumé contre, avoir pleuré contre, avoir juré contre, avoir gémi contre, lui avoir dit : non ", PP, 304) comme d'un point de vue thématique (avec, par exemple, l'éloge débonnairement lyrique du vin qui se trouve dans la bouteille, qu'on ne peut pas cacher parce qu'il « sent trop bon » et qu'il « a trop bon goût », PP, 304), le discours est aussi simple que la parole des personnages. Comme il le soutient lui-même à propos des paysans dont il fait l'éloge, l'orateur va profond sans se préoccuper «du plaisir de dessus », tout superficiel (PP, 304). La prestation de l'un des leurs à la tribune se produit dès lors avec l'accord et l'adhésion de tous - que les interventions de Bovard viennent simplement mettre en évidence. Le vigneron ne se trompe donc pas quand il prétend, parlant de l'allocution solennelle tenue devant lui, que c'est comme il a toujours dit. L'orateur, sans la moindre affectation ni d'un côté ni de l'autre, est profondément inscrit dans la communauté villageoise ${ }^{\mathrm{I} 3}$.

Le langage ramuzien est donc commun aux deux sens du terme : il l'est parce qu'il se révèle " sans distinction » et même " banal » ${ }^{14}$; mais il l'est aussi parce qu'il « s'applique [...] à toutes les personnes [...] d'un ensemble considéré ", comme le dit le Trésor de la langue française - et qu'il est donc fondamentalement partagé. Quand l'orateur propose de boire à ce qui réunit, il évoque d'abord «l'union du travail », puis « celle d'ensuite » $(\mathrm{PP}, 305)$ : c'est que toute la vie de la population se déroule dans un échange constant, celui de l'expression verbale, mais aussi celui des valeurs terriennes et d'une morale chrétienne souvent rappelée. Ainsi, bien que le discours ne soit constitué que d'idées reçues, il n'en figure pas moins le propos d'un sage - et il provoque, en dernier ressort, la cohésion de la communauté. Puisque tous ne s'expriment que par du lieu commun, chacun se reconnaît pleinement dans le discours de l'autre.

La scène du second discours est prise dans une gradation par rapport à la première partie de la section ${ }^{15}$. La deuxième allocution elle-même adopte une tournure plus sérieuse, car le nouvel orateur, lui aussi anonyme, est plus étudié que le précédent, comme le montre dès l'abord son adresse aux

I3 L'apostrophe respective des discours de Lieuvain et de l'orateur ramuzien donne d'ailleurs le ton : là où le premier s'adresse à l'assemblée par un "vous, hommes de progrès et de moralité ! » certes obséquieux, mais qui a pour effet d'établir une séparation entre son public et lui $(\mathrm{MB}, 276)$, le second dit quant à lui très sobrement : « vignerons, tireurs, chers amis » $(\mathrm{PP}, 304)$. I4 TLf, s. $v$. « commun».

I5 Chez Flaubert, au contraire, la concentration des auditeurs se perd au fil de la scène, de sorte que le texte du second orateur est seulement mentionné comme " point aussi fleuri que celui du Conseiller » mais « se recommand[ant] par un caractère de style plus positif, c'est-àdire par des connaissances plus spéciales et des considérations plus relevées » (MB, 280). Sur ce plan-là également, le narrateur ramuzien accorde donc une attention plus charitable aux propos tenus par ses personnages successifs. 
« Messieurs » qui forment l'assemblée (PP, 305). Mais les effusions du personnage qui se manifeste depuis le public sont néanmoins plus burlesques que les interventions de Bovard : le dénommé Calamin, c'est l'ivrogne du village qui, dans un chapitre précédent, titubait dans les vignes pour rentrer chez lui, tard le soir. Le contraste entre les deux intervenants est donc plus grand que dans le premier cas et la scène devient même cocasse lorsque des gens tentent de faire taire le perturbateur qui, à son tour, souhaite donner à entendre son approbation.

Du point de vue de notre problématique, le deuxième discours se révèle particulièrement intéressant, puisque le lieu commun s'y trouve ouvertement abordé par l'orateur, qui affirme en guise de préambule : «Je viens vous dire les mêmes choses [que le premier intervenant] parce qu'on s'aperçoit bientôt que les grandes choses sont en petit nombre et toujours pareilles » (PP, 305). Le nouveau tribun énonce explicitement la thèse même que j'essaie d'exposer : que si les personnages ont tendance à dire, successivement, " les mêmes choses ", «toujours pareilles ", c'est parce que les idées qu'on peut reconnaître comme " grandes » sont « en petit nombre ». Une telle grandeur, il faut l'entendre aussi bien sur un plan éthique que sur un plan esthétique. Je crois qu'on ne peut mieux dire la valeur du lieu commun que dans cette phrase.

La thématisation de cette valeur va de pair avec un nouvel accord entre les deux voix qui s'expriment : comme le premier discours ressemble aux propos réflexifs de Bovard, le second discours présente - contre toute attente - des similitudes avec l'énonciation particulière de Calamin. La scène dans laquelle l'ivrogne, assez fortement aviné, essaie de rentrer chez lui au sortir d'un café, est présentée en focalisation interne ; sur certaines portions, on pourrait même dire sans doute qu'il s'agit de style indirect libre. Sur son chemin, il rencontre par exemple une cascade, qu'il perçoit de cette manière :

On voit l'eau pendre à un de ces bancs de rocher entre des buissons et un ou deux frênes. Dans sa robe blanche, sautant sur un pied dans sa robe de mousseline toute en volants comme celles des filles le dimanche; alors il s'arrête pour elle, alors te voilà, toi ! bonjour! (PP, 270).

Bien que son style soit un peu moins fantasque, parce que plus sobre, l'orateur figure son rapport au terroir d'une manière au fond assez proche, dans la suite de son développement : " ... il y a une terre qui nous a pris contre elle, et elle est là, vous la voyez bien, toute proche, qui nous a mis sur ses genoux, nous serrant si fort qu'elle peut dans sa jupe contre sa poitrine » (PP, 306). Dans les deux tirades, le même animisme sensuel se fait jour, déployant une imagerie attentive aux formes et aux vêtements féminins. Une nouvelle fois les discours des deux intervenants de la scène tendent dès lors à se rejoindre. 
Leur coïncidence peut d'ailleurs être exprimée de manière plus fondamentale encore. Le passage qui constitue le cœur du second discours, structurellement et thématiquement, me paraît en effet construit de manière à montrer - de manière paradoxale - que le principal ne passe pas par les mots. L'orateur commence par dire : "Car le monde a mis six jours à se faire, et aujourd'hui seulement vient le septième jour... » ; puis, pendant un instant, sa parole est recouverte par l'extravagant auditeur, comme l'indique le narrateur : "Et Calamin a dit encore quelque chose en levant la main, sa voix se perd parmi l'autre voix »; alors seulement le public entend à nouveau l'orateur, dont il attrape simplement au vol que la phrase se termine par « ... Une seule personne » (PP, 305). On imagine bien qu'il a dit quelque chose comme : «tous les gens inclus dans notre communauté ne forment plus qu'une seule personne » (il redistribue d'ailleurs cette entité unique dans les « jeunes, vieux, hommes, femmes, enfants » qu' il énumère ensuite); mais on n'a aucune confirmation de cette lecture. On n'en a d'ailleurs pas besoin, parce que l'important n'est pas ce qui a été prononcé : l'important, c'est que la voix de Calamin puisse "se perd[re] parmi l'autre voix », si bien que ces deux paroles mêlées manifestent leur adéquation même quand elles ne sont pas comprises. La valeur la plus haute est ici donnée, d'une manière très nette, à la communauté, et à son union - au point qu'il ne semble pas abusif de parler désormais de communion.

À partir de ces correspondances entre les discours des deux orateurs et la parole des personnages en général, il est possible d'aller plus loin. Les diverses interventions présentent en effet toutes les caractéristiques également du style du narrateur de Passage du poète - et, disons-le tout de suite, de Ramuz lui-même - avec ses répétitions marquées, ses tournures régionales et son goût de la simplicité lexicale. À propos même d'une possibilité d'union, un extrait du second discours permet d'illustrer cette remarque :

... et il y a les sexes, il y a les âges, mais il n'y a plus rien qui sépare, étant tellement mêlés ensemble, voyez-vous, qu'on ne va plus jamais pouvoir se démêler... [...] ... Qu'on ne démêlera plus jamais ces mêmes sangs qui se sont mêlés, en ce jour, comme je voulais dire, ce septième jour... (PP, 306).

Une description assumée par le narrateur du roman présente de fortes similitudes avec ce chiasme de l'orateur entre être mêlé et ne plus pouvoir se démêler. Il s'agit d'un jeune homme, nommé Lambelet, qui est invité à rejoindre un trio d'hommes dans un café - et, pour évoquer le moment où ils en ressortent tous ensemble, Ramuz écrit :

Et Lambelet est avec eux, Lambelet est au milieu d'eux. Il n'y a plus eu de Lambelet. Il est entré dans les trois qu'ils étaient, ce qui en fait quatre, mais 
un. Ils marchent l'un à côté de l'autre, tenant toute la largeur de la route, on ne les a plus distingués; c'est la ressemblance des corps, il n'y a plus de différence et c'est la communication des cœurs et ils ne sont plus séparés... (PP, 283).

Comme le second orateur, le narrateur décrit une cohésion si étroite qu'elle autorise à ne plus prendre en considération les différences singulières : suite à un étrange phénomène arithmétique qui voit la communauté annihiler les individualités pour mieux les unir, les quatre hommes en présence ne forment plus qu'une seule personne. Ce n'est donc pas un hasard si le sujet, précisément, de « la ressemblance des corps » et de « la communication des cœurs » met aussi sensiblement d'accord les personnages et leur narrateur ${ }^{16}$ : l'auteur s'exprime partout dans les mêmes termes et selon les mêmes tournures que ses créatures romanesques, manifestant plus nettement encore la concorde qui se dégageait au moment de l'énonciation des discours fictifs.

Le langage humain, chez Ramuz, peut sans doute être qualifié de "fêlé ", comme dans une métaphore célèbre employée par le narrateur de Madame Bovary (MB, 319). Mais l'authenticité des rapports humains compense un tel défaut : la communication entre les hommes passe certes par le langage pour s'épancher, mais comme à travers lui ; et les sentiments de sympathie se transmettent malgré l'imperfection des moyens d'expression. Il importe simplement de parler, de sorte que le propos, dans son fond comme dans sa forme, peut bien demeurer commun. Le lieu commun (comme parole du bon sens partagé) et le discours médiocre (celui du personnage non cultivé, du terrien, de l'homme de condition modeste, mais qui n'en est pas moins le détenteur d'une véritable sagesse) désignent le moment où une authentique communion peut se produire.

Toute une éthique transparaît donc à travers le rapport intertextuel que Ramuz met délibérément en place avec Flaubert : il s'agit pour l'écrivain romand d'exprimer les petites gens, les « gens de métier », comme il dit ${ }^{17}$,

I6 Les bourgeois assistant aux comices, dans les Mœurs de province, sont également très semblables les uns aux autres sur le plan du physique, comme le montre la description particulièrement généralisante qui est donnée de leur groupe : "Tous ces gens-là se ressemblaient. Leurs molles figures blondes, un peu hâlées par le soleil, avaient la couleur du cidre doux, et leurs favoris bouffants s'échappaient de grands cols roides, que maintenaient des cravates blanches à rosette bien étalée. Tous les gilets étaient de velours, à châle ; toutes les montres portaient au bout d'un long ruban quelque cachet ovale en cornaline ; et l'on appuyait ses deux mains sur ses deux cuisses, en écartant avec soin la fourche du pantalon, dont le drap non décati reluisait plus brillamment que le cuir des fortes bottes » $(M B, 274)$. Mais, si une « ressemblance des corps » se révèle bien ainsi, elle n'en conduit pas pour autant, visiblement, à la « communication des cœurs ».

I7 Voir par exemple Charles Ferdinand Ramuz, "Anti-poétique », in Salutation paysanne et autres morceaux, Nouvelles et morceaux, 5 vol., Genève, Slatkine, 2006-2007, t. IV, p.307. 
en leur apprenant aussi bien à s'exprimer par eux-mêmes, ce qui constitue l'éminente fonction assignée à son " passage du poète ». L'humanisme ramuzien revendique un art passant par le discours le plus médiocre qui soit, et formé presque uniquement de lieux communs - étant entendu que commun, aussi bien que médiocre, a changé sinon de sens, au moins de valeur. Les deux adjectifs ne sont plus les emblèmes d'un ridicule ou d'une pauvreté, mais les signes d'une grandeur intrinsèque. Et la grandeur de Ramuz luimême, pour moi, elle est là : elle est de relever les simples pour montrer toute la valeur de leur parole. 